ORIGINAL ARTICLE

\title{
Diagnostic Accuracy of CT Scans in Blunt Abdomical Trauma
}

\author{
AZMAT ALI ${ }^{1}$, NADIA KHATTAK ${ }^{2}$, ADNAN AHMED $^{3}$, JAWAD ALI MEMON ${ }^{4}$, MAIMOONA AFSAR $^{5}$, HAFEEZ UR REHMAN $^{6}$ \\ ${ }^{1}$ Assistant Professor Radiology, Ayub Teaching Hospital, Abbottabad \\ ${ }^{2}$ Assistant Professor Diagnostic Radiology, Hayatabad Medical Complex, Peshawar \\ ${ }^{3}$ Associate Professor, Radiology Liaquat University of Medical and Health Sciences, Jamshoro \\ ${ }^{4}$ Assistant Professor Radiology, People's University of Medical and Health Sciences for Women (PUMHSW), Shaheed Benazir Abad \\ ${ }^{5}$ SPR Radiology, Hayatabad Medical Complex Peshawar \\ ${ }^{6}$ Senior Registrar Radiology, Gajju Khan Medical College, Swabi \\ Corresponding author: Nadia Khattak, Email: nadiaishfaq.7@gmail.com, Cell: +923339326507
}

\begin{abstract}
Background and Aim: Blunt abdominal trauma may lead to a serious situation that necessitates prompt diagnosis and treatment. The intra-abdominal injury effective diagnosis is difficult in patient's management in such cases. The present study aims to evaluate the diagnostic accuracy of computed tomography scans in active internal bleeding following abdominal trauma. Materials and Methods: This cross-sectional study was carried out on 84 blunt abdominal trauma patients in the Department of Radiology Hayatabad Medical Complex, Peshawar for duration of six months from January 2021 to June 2021. All the patients with no clinical manifestations underwent diagnostic CT scans. Those who had a positive CT scan underwent laparotomy. The remaining patients were monitored for 24 hours and were discharged in case of no serious issues. Demographic details, patient information, trauma mechanism, CT scan indications, and findings, and laparotomy results were recorded. Regarding injured organs, specificity, sensitivity, and diagnostic accuracy of CT scans were determined. For individual cases, CT scans specificity, sensitivity, and accuracy were calculated. SPSS version 20 was used for data analysis.

Results: This study included 84 patients, out of which $73(86.9 \%)$ were male and $11(13.1 \%)$ were female. Patients aged $20-40$ years were more prevalent $47(56 \%)$, followed by those aged over 40 years were $20(23.8 \%)$ and 10 to 19 years old were 17 $(20.2 \%)$. Liver and spleen injuries had the highest CT scan sensitivity of $87.5 \%$ and $96.7 \%$ respectively. On the other hand, The CT scan's specificity was high in the injured kidneys (94\%) and retroperitoneal hematoma 97.3\% compared to other organ's injuries. The diagnostic accuracy of CT scans in the identification of liver, kidney injuries, spleen, and retroperitoneal hematoma injuries was $93.7 \%, 92.3 \%, 96.2 \%$, and $92.3 \%$ respectively.

Conclusion: Our study found that CT scans are capable of detecting a wide range of injuries. CT scans are exceptionally valuable for assessing blunt abdominal injuries with active internal bleeding patients Furthermore, CT investigation is quick and extensively accessible. CT can produce high-resolution images with MPR and scanning protocol.

Keywords: Diagnostic Accuracy; Abdominal Trauma Injuries, Computed Tomography (CT)
\end{abstract}

\section{INTORDUCTION}

Rapid recognition of lethal injuries and appropriate care quick initiation may improve the survival chances for trauma patients. Nevertheless, it is frequently challenging to evaluate blunt abdominal injuries accurately, which may be obscured by visible external injuries. In thermodynamically stable patients, CT scans is the preferred imaging modality for evaluating abdominal injury [1]. CT diagnosis can facilitate a quick and precise assessment of the viscera, abdominal wall, and retroperitoneum [2]. Furthermore, an abdominal CT scan can aid in the synchronized abdominal injuries evaluation such as previously undetected pelvic and spinal fractures [3]. The CT scans capability to accomplish and generate accelerated images like multi planar reconstruction is critical for abnormality accurate interpretation. The medical literature contains a variety of studies on the efficacy and diagnostic accuracy of CT in blunt abdominal trauma evaluation; a highly discussed and has caused much argument [4-8].

CT as imaging modality shown usefulness in the solid organ injuries finding as well as revealing ongoing internal bleeding. The development of thin-section multidetector CT (MDCT) has also improved the detection of bowel and mesenteric injuries [9]. The computed tomography (CT) uses is the recent developments in solid organ injuries management instigation a superior emphasis on non-invasive treatment [10]. Although the surgical intervene decision is made on medical norms instead of image findings [11], CT information frequently improves diagnostic confidence and reduces the number of unnecessary exploratory laparotomies [12]. The use of Focused Assessment Sonography for Trauma (FAST) for unstable patients with intra-abdominal hemorrhage rather than injury has transformed the methods for diagnosing blunt abdominal trauma. CT scans are now regarded as a rapid method of assessment for the early detection of intraperitoneal injury that does not rely on the operator [13]. As a result, this study was carried out to assess the specificity and sensitivity of CT-scan findings in patients with blunt abdominal trauma in order to validate the method as a non-operator-dependent method that residents in teaching hospitals could use. Blunt abdominal trauma was assessed with CT scans conducted on 92 patients in a tertiary referral center. All the patients underwent CT scans due to suspicious injuries of the intra-abdomen. The diagnostic accuracy of CT scans was reported $72 \%$ of various organs [14]. The present study sought to assess the diagnostic accuracy of CT scans in active internal bleeding following abdominal trauma.

\section{MATERIAL AND METHODS}

This cross-sectional study was carried out on 84 blunt abdominal trauma patients in the Department of Radiology Hayatabad Medical Complex, Peshawar for duration of six months from January 2021 to June 2021. All the patients with no clinical manifestations underwent diagnostic CT scans. Those who had a positive CT scan underwent laparotomy. The remaining patients were monitored for 24 hours and were discharged in case of no serious issues. Demographic details, patient information, trauma mechanism, CT scan indications, and findings, and laparotomy results were recorded. Regarding injured organs, specificity, sensitivity, and diagnostic accuracy of CT scans were determined. For individual cases, CT scans specificity, sensitivity, and accuracy were calculated. Abdominal tenderness, visible abdominal/pelvic trauma, homodynamic instability history, abnormal pelvic x-ray, reduced mental state, hematuria, neurological injury, intubations, loss of consciousness, Injury mechanism (high speed/energy accident/extensive vehicle damage), and pelvic fractures were all indications for an abdominal CT scan. Laparotomies were performed on all patients with positive CT results and had intraabdominal and pelvic injuries evidence while others were monitored for 24 hours and discharged if no problems were reported; otherwise, they underwent laparotomy.

Data on patients' demographics, trauma mechanism, CT scan indications, CT scan findings, and laparotomy results were collected. The CT-scan image's sensitivity, specificity, and accuracy in relation to the injured organ were calculated. The sensitivity of the CT scan was defined as its ability to detect an 
injury if one existed, and it was calculated by dividing the number of true positive CT scans by the number of positive laparotomies. The specificity was defined as a CT scan's ability to rule out an injury that did not exist, and it was calculated by dividing the number of true negative CT scans by the number of patients who did not have an injury. Finally, the accuracy of the CT scan was calculated by dividing the total number of CT scans performed by the sum of true positive and true negative CT scans. SPSS version 20 was used to enter the data. The variable was examined using the Student t-test and Chi-square analysis.

\section{RESULTS}

This study included 84 patients, out of which 73 (86.9\%) were male and $11(13.1 \%)$ were female. Patients aged $20-40$ years were more prevalent $47(56 \%)$, followed by those aged over 40 years were 20 (23.8\%) and 10 to 19 years old were $17(20.2 \%)$. Liver and spleen injuries had the highest CT scan sensitivity of $87.5 \%$ and $96.7 \%$ respectively. On the other hand, The CT scan's specificity was high in the injured kidneys $94 \%$ and retroperitoneal hematoma $97.3 \%$ compared to other organ's injuries. The diagnostic accuracy of CT scans in the identification of liver, kidney injuries, spleen, and retroperitoneal hematoma injuries was $93.7 \%, 92.3 \%, 96.2 \%$, and $92.3 \%$ respectively. The prevalence of accident and fall injuries was $53(63.1 \%)$ and $19(22.6 \%)$ respectively. The age and gender-wise distribution of 84 patients are shown in Figure-1 and 2 respectively. The positive predictive value (PPV) and negative predictive value (NPV) of various organs diagnosed through CT scans are shown in Figure-3. Table- 1 shows the specificity and sensitivity of CT scans with respect to different organs injured.

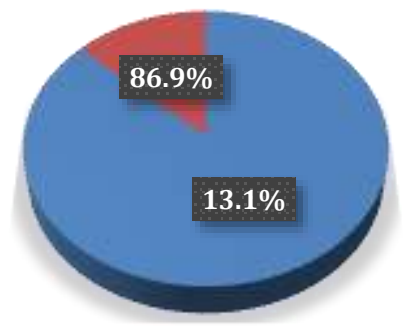

\section{Male}

- Female

Figure-1 Gender distribution $(n=84)$

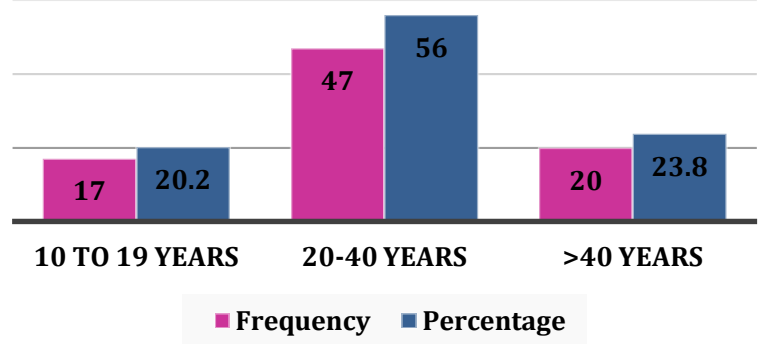

Figure-2 Age wise distribution $(n=84)$

Table-1 Specificity and Sensitivity of CT scans in various Organs

\begin{tabular}{|l|l|l|l|}
\hline $\begin{array}{l}\text { Various } \\
\text { Organs }\end{array}$ & Sensitivity \% & Specificity \% & Overall Accuracy \% \\
\hline Liver & 87.5 & 67.6 & 93.7 \\
\hline Spleen & 96.7 & 92.7 & 96.2 \\
\hline Kidney & 79.8 & 94 & 92.3 \\
\hline Stomach & 78.6 & 59.9 & 69.3 \\
\hline Pancreas & 44.8 & 81.3 & 63.1 \\
\hline Duodenum & 70.9 & 73.2 & 72.2 \\
\hline Small Intestine & 76 & 76 & 76 \\
\hline $\begin{array}{l}\text { Retroperitonea } \\
\text { I Hematoma }\end{array}$ & 64.7 & 97.3 & 92.3 \\
\hline
\end{tabular}

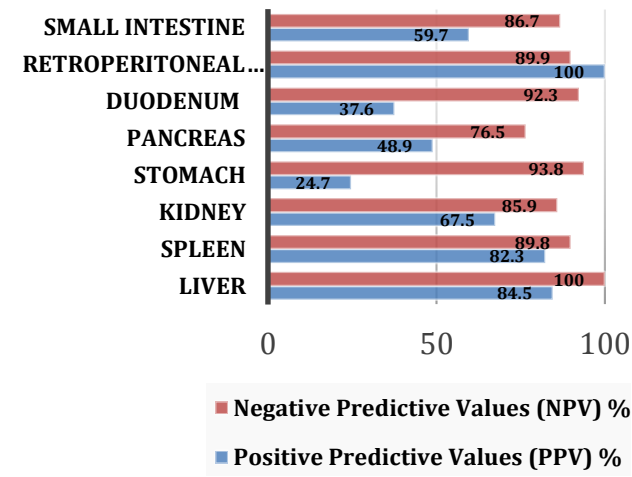

Figure-3 CT scans positive and negative predictive values

\section{DISCUSSION}

CT has proven to be an excellent imaging modality for diagnosing and managing hemodynamically stable patients with abdominal injuries over the last 20 years. 9 Sonography, on the other hand, is widely used as the preferred screening technique in many trauma centers. The utility of sonography in detecting organ injury varies greatly depending on the location of the lesion [15]. According to numerous studies, computed tomography (CT) plays an important role in the identification of suspected bowel and mesenteric injuries. It is worth noting that the accuracy of this method in diagnosing the same injuries has been called into question. The current study, on the other hand, found that CT scans were more sensitive to liver, kidney, and spleen injuries. This diagnostic method has a low sensitivity in patients with pancreas, mesentery, and colon injury, according to these findings. In a previous study, it was also discovered that $13 \%$ of patients with perforated small bowel injury had an abnormal CT scan prior to surgery [16].

Others have established CT as the gold standard for detecting retroperitoneal hematomas and kidney injuries [17]. Similarly, the current study supported the previously mentioned findings. Furthermore, many researchers believe that CT scans should be reserved for patients with negative sonographic findings and clinical suspicion of injury. In their study, Marco et al [18] reported acceptable accuracy for fluid and liver, splenic, and renal injuries in patients with major trauma. In other words, CT had a high diagnostic accuracy for single lesions in both major and minor trauma, according to the researchers. They hypothesized that the false negative reports in their study were caused by a lack of detail in whole-body protocols and artefacts caused by the patients' condition. Livingston et al [19], on the other hand, believe that patients with suspected abdominal injury should be hospitalized for at least 24 hours, even if CT scans show no signs of injury.

CT scans have a high sensitivity and specificity for detecting blood in the peritoneal cavity [20]. The formation of haemoperitoneum begins with site injury and feasts on anticipated anatomic conduits [21]. Active hemorrhage can seem like an extravagated region of dissimilarity substantial and is designated on high attenuation values of $85-350 \mathrm{HU}$ [22]. The location of distinction extravasations seen on CT imaging resembles to the bleeding location [23]. During blunt abdominal trauma, the spleen is the most commonly injured abdominal organ contributing to $45 \%$ visceral injuries $[24,25]$. It is possible to conclude that, when compared to other diagnostic methods such as ultrasonography, CT scans are particularly useful for solid organs. However, when compared to DPL, it is a less invasive method. Because CT images have a high specificity in the absence of injury, no laparotomy is required when the CT scan images are reported to be normal. The current study's findings indicate that CT scan can be a good option, particularly for patients with blunt abdominal trauma in teaching hospitals. Many people believe that because the sonography is not performed by an expert radiologist during 
these hours, it lacks acceptable accuracy. Residents can obtain images with acceptable sensitivity and specificity for reporting abdominal injuries using CT scan.

\section{CONCLUSION}

Our study found that CT scans are capable of detecting a wide range of injuries. CT scans are exceptionally valuable for assessing blunt abdominal injuries with active internal bleeding patients Furthermore, CT investigation is quick and extensively accessible. CT can produce high-resolution images with MPR and scanning protocol.

\section{REFERENCES}

1. Pediatric Trauma. In. Stewart R, Henry S, editors. ATLS student manual, 10 ed. Chicago: American College of Surgeons; 2018; 188

2. Sathya C, Alali AS, Wales PW, et al. Computed tomography rates and estimated radiation-associated cancer risk among injured children treated at different trauma center types. Injury 2019;50:1428.

3. Lodwick DL, Cooper JN, Gonzalez DO, et al. Disparities in radiation burden from trauma evaluation at pediatric versus nonpediatric institutions. J Surg Res 2018; 232:475-83.

4. Strek CJ, Vogel AM, Zhang J, et al. Identifying children at very low risk for blunt abdominal trauma in whom CT of the abdomen can be avoided safely. J Am CollSurg 2017;224:449-58.

5. Golden J, Isani M, Bowling J, et al. Limiting chest computed tomography in the evaluation of pediatric thoracic trauma. J Trauma Acute Care Surg 2016;81:271-7.

6. Halaweish I, Riebe-Rogers J, Randall A, et al. Compliance with evidence-based guidelines for computed tomography of children with head and abdominal trauma. J PedSurg 2018;53:748-51.

7. Leeper CM, Nasr I, Koff A, et al. Implementation of clinical effectiveness guidelines for solid organ injury after trauma: 10-year experience at a level1 pediatric trauma center. $J$ PedSurg 2018;53:775-9.

8. Roden-Foreman JW, Rapier NR, Foreman M, et al. Rethinking the definition of major trauma: The need for trauma intervention outperforms Injury Severity Score and Revised Trauma Score in 38 adult and pediatric trauma centers. J Trauma Acute Care Surg 2019;87:658-65. https://doi.org/10.1097/TA.0000000000002402.

9. Abbreviated Injury Scale 2005. Update. Gennarelli TA, Wodzin. E Editors Association for the Advancement of Automotive Medicine: Chicago 2008;2016(Clarification document).

10. Callahan MJ. CT dose reduction in practice. PediatrRadiol 2011;41(Suppl. 2):488-92. [14] Boylan JK. Image gently at 10 years. J Am CollRadiol 2018;15:1193-5.

11. Puffenbarger MS, Ahmad FA, Argent M, et al. Reduction of computed tomography use for pediatric closed head injury evaluation at a nonpediatric community emergency department. AcadEmerg Med 2018;26:784-95.

12. Meltzer JA, Stone ME, Reddy $\mathrm{SH}$, et al. Association of whole-body computed tomography with mortality risk in children with blunt trauma. JAMA Pediatr 2018;172: 542-9.

13. Ide K. Uematsu S, Hayano $S$, et al. External validation of the PECARN head trauma prediction rules in Japan AcadEmerg Med 2019. https://doi.org/10.1016/j.ajem. 2019.158439 [Epub ahead of print].

14. American College of Surgeons Trauma Quality Improvemen Program (ACS TQIP) best practices guidelines in imaging. October 2018. 17-8. Available at https:// www.facs.org/ /media/files/quality\%20programs/trauma/tqip/imaging guidelines. ashx. Accessed date: 20 June 2019.

15. Arbabi CA, Vogel AM, Plumblee $L$, et al. External validation of a fivevariable clinical prediction rule for identifying children at very low risk for intra-abdominal injury after blunt abdominal trauma. J Trauma Acute Care Surg 2018;85:71-7.

16. Drucker NA, McDuffie L, Groh E, et al. Physical examination is the best predictor of the need for abdominal surgery in children following motor vehicle collision. J Emerg Med 2018;54:1-7.

17. Miglioretti DL, Johnson E, Williams A, et al. The use of computed tomography in pediatrics and the associated radiation exposure and estimated cancer risk. JAMA Pediatr 2013; 167:700-7.

18. Arbra CA, Vogel AM, Plumblee $L$, et al. External validation of a fivevariable clinical prediction rule for identifying children at very low risk for intra-abdominal injury after blunt abdominal trauma. J Trauma Acute Care Surg. 2018;85(1):71-7.

19. Arbra CA, Vogel AM, Zhang J, et al. Acute procedural interventions after pediatric blunt abdominal trauma: a prospective multicenter evaluation. J Trauma Acute Care Surg. 2017;83(4):597-602.

20. Sathya C, Alali AS, Wales PW, et al. Computed tomography rates and estimated radiation-associated cancer risk among injured children treated at different trauma center types. Injury. 2019;50(1):142-8.

21. Streck CJ, Vogel AM, Zhang J, et al. Identifying children at very low risk for blunt intra-abdominal injury in whom CT of the abdomen can be avoided safely. J Am Coll Surg. 2017;224(4):449-58 [e3]

22. Goodman TR, Mustafa A, Rowe E. Pediatric CT radiation exposure: where we were, and where we are now. PediatrRadiol. 2019;49(4):469-78

23. Holmes JF, Lillis K, Monroe D, et al. Identifying children at very low risk of clinically important blunt abdominal injuries. Ann Emerg Med. 2013;62(2):107-16 [e2].

24. McGrew PRCP, Fisher JD, Kuhls DA, et al. Implementation of a CT scan practice guideline for pediatric trauma patients reduces unnecessary scans without impacting outcomes. J Trauma Acute Care Surg. 2017;85(3):45-8.

25. Vogel AM, Zhang J, Mauldin PD, et al. Variability in the evaluation of pediatric blunt abdominal trauma. PediatrSurg Int. 2019;35(4):479 85. 